\title{
Visual mismatch negativity (vMMN) for low- and high-level deviances: A control study
}

\author{
Domonkos File ${ }^{1,2,3} \cdot$ Bálint File $^{4,3} \cdot$ Flóra Bodnár ${ }^{1,2,3}$ - István Sulykos ${ }^{1,2,3}$ • \\ Krisztina Kecskés-Kovács ${ }^{3}$. István Czigler ${ }^{3}$
}

Published online: 14 July 2017

(C) The Psychonomic Society, Inc. 2017

\begin{abstract}
The aim of our studies was to separate the effects of violating a sequential rule (genuine visual mismatch negativity; gvMMN) from the decreased activity in response to repeated stimuli (stimulus-specific adaptation; SSA) for simple and more complex stimuli. To accomplish this goal, different control procedures were applied with the aim of finding the correct control for vMMN studies. Event-related brain electric activity (ERPs) was measured in response to nonattended visual stimuli that were presented either in an oddball manner or in various control sequences. To identify the cortical sources of the different processes, the SLORETA inverse solution was applied to the average ERP time series. In Experiment 1, the stimuli were line textures, and the deviancy was different line orientations. SSA fully explained the deviant-related ERP effects (increased posterior negativity in the 105-190 ms range). In Experiments 2 and 3, windmill patterns were used. Infrequent windmill patterns with 12 vanes elicited gvMMN (posterior negativities in the 100-200 and 200-340 ms ranges), whereas
\end{abstract}

Domonkos File

file.domonkos@ttk.mta.hu

1 Department of Cognitive Psychology, Eötvös Loránd University, Faculty of Education and Psychology, Budapest, Hungary

2 Doctoral School of Psychology, Eötvös Loránd University, Budapest, Hungary

3 Research Centre of Natural Sciences, Institute of Cognitive Neuroscience and Psychology, Cognitive Psychology Group, Hungarian Academy of Sciences, Budapest, Hungary

4 Faculty of Information Technology and Bionics, Pazmany Peter Katolikus Egyetem, Budapest, Hungary in the case of the less complex (six vanes) stimuli, SSA explained the negative deflection in both latency ranges (178-216 and 270-346 ms). In Experiment 3, infrequent stimuli with six vanes elicited deviant-related posterior negativity within the sequence of less complex (four vanes) frequent patterns. We reconcile the discrepant results by proposing that the underlying processes of vMMN are not uniform but depend strongly on the eliciting stimulus and that the complexity difference between the infrequent and frequent stimuli has considerable influence on the deviant-related response.

Keywords Evoked potentials · Visual perception

Change blindness studies have shed light on the poor ability of humans to explicitly detect changes between two successive visual images that are separated by a blink or saccade (Simons \& Levin, 1997). Traditional interpretation of the phenomenon states that representations outside the focus of attention are volatile, thus focal attention is necessary to detect changes in the visual environment (Rensink, 2002). However, an increasing body of studies shows that the human brain is capable of detecting even small changes, especially if such changes violate automatic (nonconscious) expectations based on repeating experiences (Stefanics, Kremlacek, \& Czigler, 2014). Over the past 15 years, many studies have demonstrated that unattended visual stimuli that violate the rules of a stimulus sequence (deviants) elicit larger responses in event-related brain activity than regular (standard) stimuli. The difference between the standard and the deviant stimuli is called visual mismatch negativity (vMMN), which is considered to be the visual homolog of the auditory mismatch negativity (MMN) component of event-related potentials 
(ERPs; for reviews, see Czigler, 2007; Kimura, 2012; Stefanics et al., 2014). The traditional paradigm in the field of vMMN research is the passive oddball paradigm, in which task-unrelated stimulus sequences of infrequent (deviant) and frequent (standard) stimuli are presented.

The ERP difference between the effects of the deviant and standard stimuli can be either the consequence of an activity decrease in response to the standards over the sequence or an additional activity elicited by the deviants. An activity decrease in response to repeated stimuli is a well-known effect at each level of brain activity, from single cell recording (Sawamura, Orban, \& Vogels, 2006) to conscious experience (Clifford, 2002; Gibson, 1937; Krekelberg, Boyton, \& Wezel, 2006) and has been labelled as refractoriness, habituation, or stimulus specific adaptation (SSA). ${ }^{1}$ A few explanations have attempted to attribute the whole deviant-minus-standard difference as a repetition-related activity decrease of the standard response (e.g., May \& Tiitinen, 2010, in the auditory modality; Kenemans, Jong, \& Verbaten, 2003, in vision). However, most theories explaining MMN, in addition to the repetition-related response decrement, are assuming a process attributed to the novel stimuli. Winkler, Karmos, and Näätänen (1996) proposed a model-adjustment account, which states that the MMN reflects online modifications of a perceptual model (see also Czigler, 2007, for vMMN). On a functional level, the model predicts the forthcoming stimulation, and updating such a predictive model is necessary when the incoming stimulus does not match the predicted stimulus (Friston, 2005; Garrido, Kilner, Stephan, \& Friston, 2009). This model was extended to the visual MMN; the successive visual stimulation is extracted into an abstract sequential rule, which is encoded as a prediction for the forthcoming visual events (Friston, 2003, 2005; Garrido et al., 2009; Kimura, 2012; Stefanics et al., 2014; Winkler \& Czigler, 2012).

Although the relationship of the adaptation and prediction theories has remained an unsettled issue, researchers in the field have attempted to develop methods for separating repetition-related effects from deviant-related additional activity. The most frequent method of separating the two sources of difference is equal probability control (in the auditory modality: Jacobsen \& Schroger, 2001; Schröger \& Wolff, 1996; in vision: Amando \& Kovács, 2016; Astikainen, Cong,

\footnotetext{
${ }^{1}$ There is no consensus with regard to the term that describes the response attenuation due to stimulus repetition. In MMN research, the most common term is refractoriness, as indicated by O'Shea (2015). However, as O'Shea highlights, the physiological meaning of refractory reflects the inactive state of a neuron due to previous electrical activity, which is on the order of milliseconds and is much too short to be the presumed process that underlies MMN. Furthermore, when considering that other terms are often in use as synonyms, O'Shea suggests the use of the term adaptation (O'Shea, 2015). We will use his suggestion (i.e., the term adaptation).
}

Ristaniemi, \& Hietanen, 2013; Astikainen, Lillstrang, \& Ruusuvirta, 2008; Czigler, Balázs, \& Winkler, 2002; Kimura, Katayama, Ohira, \& Schröger, 2009). Within the equal probability sequence, stimuli physically identical to the oddball's deviant are embedded in the sequence of other equiprobable stimuli. Within such a sequence, the probability of each stimulus type is equal to the probability of the oddball deviant. Thereafter, the ERPs to the oddball deviant and the equal probability control stimuli are compared. In the control sequence, there is no way of developing a strong memory representation (or a regularity-related memory representation) for any of the stimuli, and therefore, no additional activity is expected within the ERPs that are elicited by the equivalent control. A critical feature of the equal probability control paradigm is that the average physical separation between the control and other equiprobable stimuli is equal or larger than between the standard and deviant stimuli. This excludes the possibility of a response decrement to the control stimuli relative to the deviant due to the additional activations of the afferent neurons responding to control stimuli by other equiprobable stimuli (Jacobsen \& Schroger, 2001; Kimura et al., 2009). The difference between the activity elicited by the deviant and the control is called genuine MMN (gMMN) - that is, an additional activity without the involvement of adaptation effects. In the auditory modality, the emergence of gMMN was frequently demonstrated (e.g., Jacobsen \& Schroger, 2001; Jacobsen, Schröger, Horenkamp, \& Winkler, 2003; Ruhnau, Herrmann, \& Schröger, 2012; Schröger \& Wolff, 1996).

In the following, a brief summary is given on vMMN studies' applied control sequences. In the case of color deviancy, Czigler et al. (2002) obtained similar deviant-minus-standard and deviant-minus-control difference potentials in an early, 120-160 ms range. Pazo-Alvarez, Amenedo, and Cadaveira (2004) obtained similar differences for motion direction, again in an early (145 to $165 \mathrm{~ms}$ ) range. In a recent MEG study with spatial frequency deviants, Susac, Heslenfeld, Huonker, and Supek (2013) recorded differences in the 100-160 ms range and reported distinct localization for the gvMMN and the deviant-minus-standard difference. Facial emotion vMMN was investigated in two studies. $\mathrm{Li}, \mathrm{Lu}$, Sun, Gao, and Zhao (2012) obtained early onset ( $100 \mathrm{~ms})$ of both deviant-minusstandard and deviant-minus-control differences, both in an early (100-200 ms) and later (200-350 ms) range. Importantly, in the early range, the amplitude of gvMMN was smaller than the deviant-minus-standard difference wave. However, Astikainen et al. (2013) obtained different scalp distributions between the oddball (bilateral) and the equal probability (right-dominant) conditions in the earlier range (peaking at $130 \mathrm{~ms}$ ). In the later component of the difference potential, peaking at $170 \mathrm{~ms}$, no such difference was observable between the conditions. Orientation deviancy was investigated by Astikainen et al. (2008) and Kimura et al. (2009). In 
both studies, the stimuli were single bars. Astikainen et al. (2008) obtained negativities in the oddball and control comparisons in similar ranges (185-205 ms), but the surface distribution of the gvMMN was narrower. In the study reported by Kimura et al. (2009), however, the application of equal probability control eliminated the difference in the range that corresponded to a posterior negativity (N1), but in a later latency range (200-250 ms) gvMMN emerged. Kimura, Ohira, and Schröger (2010) reported similar results. Kimura and Takeda (2013) investigated orientation-related vMMN in response to a pattern that consisted of eight lines around the center of the visual field (the location of a size-discrimination task). Again, the use of equal probability control eliminated the early part of the response but preserved a later difference as gvMMN. On the basis of these results, Kimura et al. (2009) argued that in vision, an early, standard-related process is followed by a memory-related mismatch process, and the later process is a correlate of a predictive mechanism.

As described above, vMMN does not have a uniform latency range. In many studies, complex or higher-order ${ }^{2}$ deviances elicit a temporary wide (from approximately 100 to 350 ms) two-component vMMN (faces: Astikainen \& Hietanen, 2009; Csukly, Stefanics, Komlósi, Czigler, \& Czombor, 2013; Kecskés-Kovács, Sulykos, \& Czigler, 2013a; Li et al., 2012; Stefanics et al., 2012; Wang, Miao, \& Zhao, 2014; Zhao \& Li, 2006; categorical stimuli: Kecskés-Kovács, Sulykos, \& Czigler, 2013b; Wang et al., 2013). In contrast, simple deviances such as orientation, color, or motion direction often elicit a temporarily restricted, one-component negativity between 100 and $200 \mathrm{~ms}$ post stimulus (e.g., orientation: Czigler \& Sulykos, 2010; Sulykos \& Czigler, 2011; Takács, Sulykos, Czigler, Barkaszi, \& Balázs, 2013; color: Czigler et al., 2002; motion direction: Pazo-Alvarez et al., 2004). It is important to note that this observation cannot hold true for all vMMN studies; for example, Kimura et al. (2009) recorded twocomponent $\mathrm{VMMN}$ in response to orientation change in the 100-250 ms latency range. A possible explanation for the discrepancy is that in studies that reported long-lasting negativities for orientation deviances, either single bars (Astikainen et al., 2008; Astikainen, Ruusuvirta, Wikgren, \& Korhonen, 2004; Kimura et al., 2009; Kimura et al., 2010) or a frame-like pattern of bars (Kimura \& Takeda, 2013) were presented, whereas in studies that reported single negativities, line textures were presented (Czigler \& Sulykos, 2010; Sulykos \& Czigler, 2011; Takács et al. 2013). Also, among one dimension of deviance, the characteristics of the deviant-

\footnotetext{
${ }^{2}$ Higher/lower order/level, simple/complex stimuli are often mentioned in cognitive studies; however, as far as we know, a strict definition is lacking. In this study, high- and low-level deviances refer to the distinct dimensions of the deviance; low level is a unidimensional deviance, while high level is a multidimensional deviance. Complexity refers to the number of interconnected parts that build up the stimuli. In this sense, we use complexity as a relative term to describe the relationship between stimuli.
}

related response could vary as a function of stimulus properties. Heslenfeld (2002) found that an early refractoriness (60$100 \mathrm{~ms}$ ) effect was present only for high spatial frequencies, whereas a negative response in the 120 to $160 \mathrm{~ms}$ range was present for both low- and high-frequency deviants, followed by a later (160-200 ms) component of the difference.

The aim of the present study was to investigate the relationships between the gvMMN and the repetition-related decrease of the ERP activity to the standard stimuli for both lowand high-level stimuli. To accomplish this goal, different control procedures were applied, with the aim of finding the right control for vMMN studies. Two types of deviancies were used. In Experiment 1, orientation deviancy was investigated in a texture-like arrangement because orientation deviancy has been studied in a fairly large body of studies; some of them also applied equal probability control. We investigated whether equal probability control could eliminate the expected early difference within the 100-200 ms range. In this study, we introduced an additional control procedure. As Ruhnau et al. (2012) noted, the equal probability control procedure eliminates the sequential rule of the oddball stimuli (identical stimuli follow each other), not only the effect of a particular physical feature. Sulykos (2017) argues that standard stimuli in the oddball sequence might be accompanied by specific neural response to stimulus match. In this way, the equal probability control sequence overcontrols the oddball sequence, since no stimulus match is present for the control stimuli. Thus, the standard and the control stimuli differ not only by their probability but also by their predictability. Addressing this problem, Ruhnau et al. (2012) developed a new procedure that used equal probabilities, but the various stimuli were presented in regular sequences. We applied this procedure - the cascade paradigm - in the visual modality.

The other type of stimulus was the windmill pattern (Experiment 2), which was introduced by Maekawa et al. (2005) into vMMN research. These authors obtained two distinct deviant-minus-standard difference components in two subsequent latency ranges. We assumed that the two subcomponents might reflect different processes, SSA and gvMMN, in this way allowing us to precisely separate them with the use of equal probability control. Similar to Experiment 1, we introduced a new control paradigm (modified control) that was adapted from auditory MMN experiments (Jacobsen et al., 2003). The application of this paradigm was driven by practical and theoretical consideration. From methodological aspects, it is important to know whether is it possible to correctly estimate the amplitude of the gvMMN without presenting all contextual stimuli with equal probability in the control sequence. The theoretical motivation was to investigate the effects of putative response overlap for the incoming stimuli. Considering the tuning curves of sensitivity of the afferent neurons in the primary visual area, similar stimuli are expected to stimulate partly overlapping neuron populations, which 
leads to higher levels of adaptation in the sensory neurons, comparing to when diverse stimuli are presented. Since the physical difference of contextual and control stimuli are smaller in the modified control than in the equal probability control (see Fig. 4), the overlap is expected to be larger in the modified control compared to the equal probability control. Using an additional condition in which the supposed level of adaptation is different might serve for a better spatial and temporal localization of the effect of decreased responsiveness in the deviant-minus-standard difference wave.

The structure of Experiments 1 and 2 are the same, and three types of comparisons were made: (1) deviant-minusstandard, (2) deviant-minus-equal probability control, and (3) deviant-minus-cascade in Experiment 1 and deviantminus-modified control in Experiment 2. For identifying the cortical sources of different processes, the sLORETA inverse solution was applied on the average ERP time series.

An additional experiment was conducted to clarify the results of Experiment 2. In Experiment 3, windmill patterns were presented in oddball sequences, which is partly a replication of Experiment 2.

\section{Experiment 1}

In Experiment 1, the deviancy effects of orientation change are investigated using textures that consist of tilted bars. The stimuli were presented to the lower half of the visual field, and the task required central fixation. The task was a simple video game (Sulykos, Kecskés-Kovács, \& Czigler, 2015). Four conditions were used: two oddball sequences, an equal probability control, and the cascade paradigm (Ruhnau et al., 2012). Our aim was to determine the extent of the adaptation effect on the ERPs that were elicited in an oddball sequence.

\section{Method}

\section{Participants}

Fifteen volunteers (eight women; mean age: $23.26 ; S D=0.48$ years) participated in the study for monetary compensation. They had no ophthalmologic or neurological abnormalities. Written informed consent was obtained from all of the participants prior to the experimental procedure. The study was conducted in accordance with the Declaration of Helsinki and was approved by the Joint Committee of Ethics of the Psychology Institutes in Hungary.

\section{Stimuli and experimental design}

The four conditions consisted of bar patterns that had different orientations. Figure 1 illustrates the stimuli and their probabilities. During the experiment, a pool of 11 stimulus orientations was used $\left(26^{\circ}, 46.57^{\circ}, 62^{\circ}, 67.14^{\circ}, 87.7^{\circ}, 98^{\circ}, 108.28^{\circ}, 134^{\circ}\right.$, $\left.149.42^{\circ}, 170^{\circ}\right)$. The presented stimuli appeared against a black background (bar luminance of $36.67 \mathrm{~cd} / \mathrm{m}^{2}$ ). The visual angle of the individual bars was $1.26^{\circ}$ (length) $\times 0.088^{\circ}$ (width) from a viewing distance of $1.2 \mathrm{~m}$. The bars were presented as a texture, which consisted of three rows and seven columns, and thus, a total of 21 identical stimuli were presented to the lower two-thirds of the screen (17-in. Samsung SyncMaster 740B, $60 \mathrm{~Hz}$ refreshing rate). The stimulus duration was $100 \mathrm{~ms}$, and the average interstimulus interval (ISI) was $500 \mathrm{~ms}$ (range: $450-550 \mathrm{~ms}$, even distribution). The ISI was sufficiently long to prevent any type of motion percept.

During the oddball conditions, standards were presented with a probability of $87.5 \%$ and deviants with a probability of $12.5 \%$. In one of the oddball conditions, $170^{\circ}$ orientation stimuli served as the standard stimuli, and $26^{\circ}$ orientation stimuli served as the deviant (Oddball 1). In the reverse oddball condition, the $26^{\circ}$ orientation stimuli were the deviants (Oddball 2). Between two deviant stimuli, a minimum of four and a maximum of 10 standard stimuli were presented.

During the equal probability control condition, eight different stimuli were presented, each with the probability of $12.5 \%$ $\left(26^{\circ}, 46.57^{\circ}, 67.14^{\circ}, 87.7^{\circ}, 108.28^{\circ}, 128.85^{\circ}, 149.42^{\circ}, 170^{\circ}\right)$. Two identical stimuli never occurred successively. The difference between the standard and the deviant was $36^{\circ}$, while the minimum difference between the control and contextual stimuli was $20.5^{\circ}$. However, due to the random presentation, the average difference was greater than $36^{\circ}$.

During the cascade control paradigm, a regular sequence of five different stimuli was presented, which formed a chain of rising and falling microsequences $\left(26^{\circ}, 62^{\circ}, 98^{\circ}, 134^{\circ}, 170^{\circ}\right.$, $134^{\circ}, 98^{\circ}$, etc.). The control stimuli (which were identical to the deviant stimulus of the oddball condition) were at the ends

\begin{tabular}{|c|c|c|c|c|c|c|c|c|c|c|c|}
\hline & $26^{\circ}$ & $46.67^{\circ}$ & $62^{\circ}$ & $67.14^{\circ}$ & $87.7^{\circ}$ & $98^{\circ}$ & $108.28^{\circ}$ & $128.85^{\circ}$ & $134^{\circ}$ & $149.42^{\circ}$ & $170^{\circ}$ \\
\hline & & & & & & & & & & & \\
\hline Oddball 1 & 126 & - & - & - & - & - & - & - & - & - & 882 \\
\hline Oddball 2 & 882 & - & - & - & - & - & - & - & - & - & 126 \\
\hline Equal probability control & 126 & 126 & - & 126 & 126 & - & 126 & 126 & - & 126 & 126 \\
\hline Cascade control & 126 & - & 252 & - & - & 252 & - & - & 256 & - & 126 \\
\hline
\end{tabular}

Fig. 1 Stimuli and their probabilities of Experiment 1 (times/experiment) in the oddball and control sequences 
of the microsequences, and their probability of occurrence was the same as the deviants'.

All of the conditions totaled 1,008 stimuli, which were presented in three separated blocks in a random order.

\section{Task}

To provide sufficient control of the participants' attention, the participants played a video game presented in the upper third of the screen while stimuli were presented in the lower twothirds of the screen (see Fig. 2). The participants had to control the movements of a spaceship to be able to avoid/catch certain approaching spaceships (one at a time). The task required focused attention on the location where the approaching spaceships appeared. For more details, see Sulykos et al. (2015).

\section{Recording and measuring the electrical brain activity}

The electroencephalographic activity was recorded (DC$70 \mathrm{~Hz}$; sampling rate, $1000 \mathrm{~Hz}$; Brain Vision recording system) with active electrodes placed at 64 locations according to the extended 10-20 system, using an elastic electrode cap (Acti-Cap). The online reference electrode was at $\mathrm{FCz}$, and then, the activity was rereferenced offline to the electrode on the nose tip. Horizontal electrooculographic activity was recorded with a bipolar configuration between the electrodes that were positioned lateral to the outer canthi of the eyes. The vertical eye movement was monitored with a bipolar montage between the electrodes that were placed above and below the right eye. The impedance of the electrodes was kept below $10 \mathrm{k} \Omega$.
EEG signals were filtered offline $(0.1-30 \mathrm{~Hz}, 24 \mathrm{~dB}$ slope $)$. Epochs of $500 \mathrm{~ms}$, starting from $100 \mathrm{~ms}$ before the stimulus onset, were averaged separately for the standards, deviants and control stimuli. Trials with an amplitude change that exceeded $+/-100 \mu \mathrm{V}$ on any channel were rejected from further analysis.

Only the responses from the standard preceding a deviant were included in the standard-related average ERPs, and orientations were not investigated separately. Three types of grand averaged differences were calculated: deviant-minus-standard (OddDiff), deviant-minus-equal probability Control (EQControlDiff), and deviant-minus-cascade control (CascDiff). The differences in these comparisons cannot be explained by stimulus-specific features because the standard, deviant, and control stimuli had the same physical characteristics.

Based on previous vMMN studies (for review, see Czigler 2007), we expected the emergence of a deviant-minus-standard difference wave over the posterior electrode locations. To reinforce this expectation, we defined an electrode matrix, where the deviant-minus-standard difference wave differed from zero in the negative direction in at least 30 consecutive significant data points $(p<.01)$, based on the results of a point-by-point $t$ test applied on the whole scalp location. A $2 \times 3$ matrix of electrodes (PO3, POz, PO4, O1, Oz, O2) met this criterion, which consisted of two rows (anterior, posterior) and three columns (left, middle, right). However, because no laterality effects were present, $F(2,28)=0.30, p=.74$, to obtain easier traceability, two regions of interest were formed: parietooccipital (PO4, POz, PO3) and occipital (O2, Oz, O1).

The amplitude values of the difference waves were calculated by averaging the amplitude values of all of the data points, which consisted of the sections defined by the pointby-point $t$ test on the difference waves.

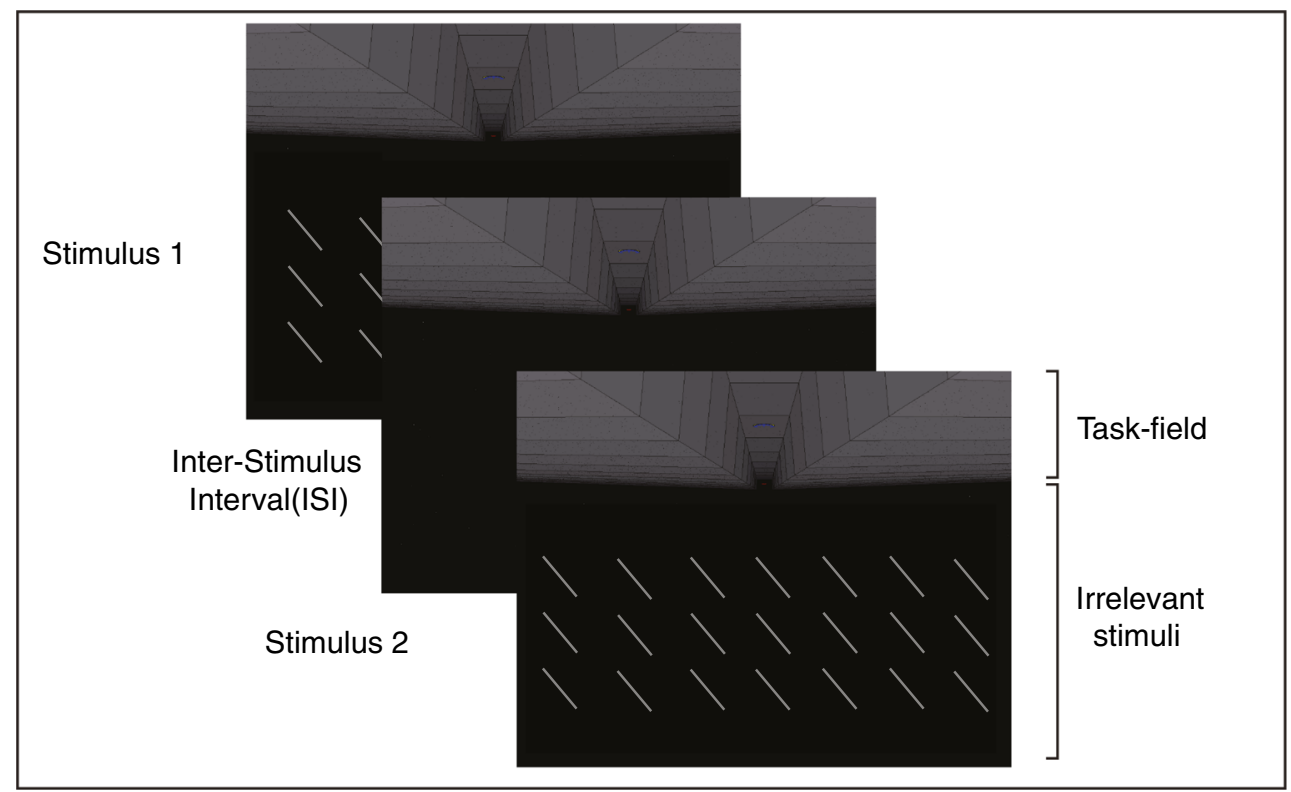

Fig. 2 Example of the stimulus display of Experiment 1 


\section{Results}

\section{Behavioral results}

The average avoidance rate was $79 \%$, while the average hit rate was $67 \%$; thus, the average performance was $72.82 \%$ (standard error of the mean; $S E M=4.7 \%$ ). There was no difference in the performance between the conditions.

\section{Event-related potential results}

Figure 3 shows the ERPs and the difference potentials. The standard, deviant and control stimuli elicited a positive (P1)negative (N1)-positive (P2) triphasic complex (see Fig. 3a).

The deviant-minus-standard difference differed from zero, $t(14)=-4.3, p<.01$, at the parieto-occipital ROI in the $105-$ $190 \mathrm{~ms}$ and in the 118-148 ms range at the occipital ROI, $t(14)=-2.98, p<.01$. Neither the deviant-minus-equal probability control nor the deviant-minus-cascade control difference waves differed from zero, and the deviant-minus-control difference waves did not differ from each other in the given range. At the parieto-occipital region in the 105-190 ms range there was a significant difference between the amplitude values of the deviant-minus-standard $(M=-0.69, S D=0.61)$ and deviant-minus-cascade control $(M=0.04, S D=0.56)$; $t(14)=-3.59, p<.01$, and for deviant-minus-standard and deviant-minus-equal probability control $(M=-0.10, S D=$ $0,34) ; t(14)=-4.60, p<.01$. Also at the occipital region, in the 118-148 ms range there was a significant difference in the amplitude values of the deviant-minus-standard $(M=-0.43$, $S D=0.56)$ and deviant-minus-cascade control $(M=0.25, S D$ $=0,73) ; t(14)=-2.67, p<.01$, and for deviant-minus-standard and deviant-minus-equal probability control $(M=-0.04, S D=$ $0,39) ; t(14)=-2.75, p<.01$. An ANOVA with the factors conditions (OddDiff, EQControlDiff, CascDiff) and ROI (parieto-occipital, occipital) revealed no latency differences between the difference waves. Table 1 shows the amplitude and latency values of the difference potentials.

The results of Experiment 1 did not support the memorycomparison-based change detection accounts because the deviant-minus-control difference and the deviant-minuscontrol differences were significantly different, and also the deviant-minus-control differences did not differ from zero in the latency range defined by the difference of the deviantminus-standard difference wave.

\section{Experiment 2}

In Experiment 2, windmill patterns were used. In studies by Kimura et al. (2009) and Kimura and Takeda (2013), the equal probability control divided the long-lasting deviant-minusstandard difference potential into two parts. In the earlier temporal window, the control procedure eliminated the difference, whereas in the later latency window, gvMMN emerged. In studies with windmill patterns (e.g., Maekawa et al., 2005), two distinct difference potentials appeared, an earlier (150$200 \mathrm{~ms}$ ) and a later (200-300 ms) component. We expect that the emergence of the first component of the difference wave is a consequence of adaptation (SSA) and the emergence of the latter component is a consequence of gvMMN.

To test this hypothesis, in addition to the oddball sequences, we applied three additional (control) sequences: an equal probability (E-control) sequence and two modified control (M-control) sequences. In the M-control sequences, the probabilities of the control stimuli were equal to that of the deviant $(p=.1)$, but in contrast to the E-control sequence, the probabilities of the individual contextual stimuli were higher than the probability of the deviant ( $p=.225)$.

It is important to note that the deviant and standard windmill stimuli have different numbers of vanes, which leads to multidimensional deviances; the larger the number of vanes, the larger the contribution of high spatial frequencies, and windmills that have a larger number of vanes can be considered to be more complex stimuli. However, we must emphasize that in this study we did not intend to analyze the effect of a specific feature per se but the effect of adaptation versus deviant-related additional activity on vMMN.

\section{Method}

\section{Participants}

Twenty-three volunteers participated in the study for monetary compensation or course credit. They had no ophthalmologic or neurological abnormalities. Written informed consent was obtained from all of the participants prior to the experimental procedure. The study was conducted in accordance with the Declaration of Helsinki and was approved by the Joint Committee of Ethics of the Psychology Institute in Hungary. One out of 23 participants was omitted from the data analyses due to the low signal-to-noise ratio, and thus, we report the data from 22 participants (14 women; mean age, 21.94; standard deviation, 1.61 years).

\section{Stimuli and experimental design}

To apply similar stimulation to the studies by Maekawa et al. (2005), but to introduce a more stringent control of attention, the participants performed a tracking task similar to the one introduced by Heslenfeld (2002), but with one level of difficulty. In the tracking task, the participants were asked to keep a ball within a blue circle $\left(1.79^{\circ}\right.$ diameter from the $120-\mathrm{cm}$ viewing distance) located at the center of the screen (i.e., within the windmill pattern). The ball was moving on the horizontal axis, with a pseudorandom speed and direction. If the ball 


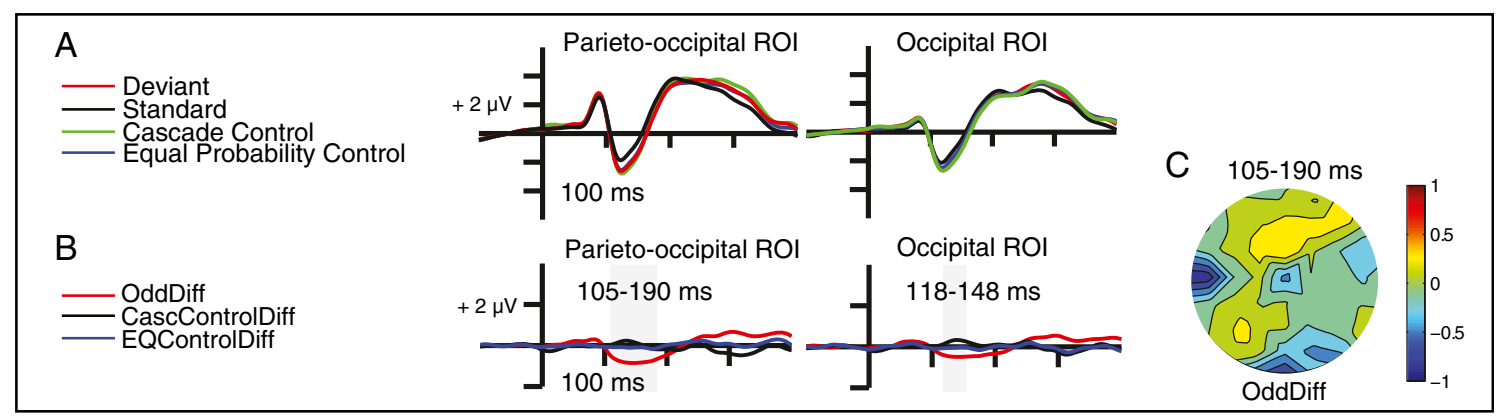

Fig. 3 Experiment 1: a Grand-averaged ERPs elicited by deviant, standard, equal probability control, and cascade control. b Grandaveraged deviant-minus standard, deviant-minus-equal probability control and deviant-minus-cascade control difference waves. c Topographical maps of the grand-averaged difference waves within the 108-208 ms time windows. (Color figure online) left the circle, the color of the circle changed from blue to red. The ball movement was controlled by a gamepad. At the end of each block performance, feedback was provided (duration within the circle/duration of the block * 100). This task required continuous central fixation, but in the case of proper fixation, a high performance was expected. The stimuli appeared on a 17-inch CRT monitor with a $60-\mathrm{Hz}$ refresh rate. The stimulus duration was $200 \mathrm{~ms}$, and the average interstimulus interval (ISI) was $800 \mathrm{~ms}$ (range: $750-850 \mathrm{~ms}$, even distribution). ERPs were recorded to windmill patterns. As Fig. 4 shows, the patterns consisted of six to 33 vanes. The patterns had high contrast $\left(37.21 \mathrm{~cd} / \mathrm{m}^{2}\right.$ for the bright and $0.14 \mathrm{~cd} / \mathrm{m}^{2}$ for the dark segments). The diameter of the pattern was $13.82^{\circ}$, and the background was gray $\left(15.96 \mathrm{~cd} / \mathrm{m}^{2}\right)$.

Five conditions were applied; Fig. 4 illustrates the stimuli and their probabilities within the conditions. The conditions were as follows (S: standard; D: deviant, and the numbers are the vanes of the patterns): Oddball S6D12, Oddball S12D6 (reverse control paradigm), equal probability control (Econtrol) and two modified control (M-control). In the oddball condition, the probability of the standard was $90 \%$ (10\% for the deviant). In each sequence, either the V6 or V12 stimuli were the standards/deviants. Between two deviant stimuli, a minimum of seven and a maximum of 12 standard stimuli were presented.

In the E-control sequence, 10 different stimuli were presented with equal (10\%) probability (V6, V9, V12, V15, V18, V21, V24, V27, V30, and V33). Two identical stimuli never occurred successively.

In the V6 M-control condition, five different stimuli were presented randomly. Four stimuli (V9, V12, V15, V18) had equal probabilities (22.5\%), and one (V6) had the probability of $10 \%$. In the V12 M-control, four stimuli (V15, V18, V21, V24) had equal probabilities of $22.5 \%$, and one stimulus (V12) had a $10 \%$ probability.

Each condition consisted of 960 stimuli, which were presented in six blocks (30 blocks in total). The blocks were presented in a semirandom order; the 30 blocks were separated into six fragments that each contained one block from every condition, in a random order.

\section{Recording and measuring the brain's electric activity}

The EEG was recorded (DC-30 Hz, sampling rate $500 \mathrm{~Hz}$; Synamps 2 amplifier, NeuroScan recording system) with Ag/ $\mathrm{AgCl}$ electrodes at 61 locations according to the extended 10 20 system using an elastic electrode cap (EasyCap). The tip of the nose was used as a reference, which was off-line

Table 1 Experiment 1: Grand averages of peak latencies and mean epochs of the difference waves measured at parieto-occipital and occipital ROIs.Values are listed in mean \pm standard error

\begin{tabular}{llll}
\hline Region & Difference waves & $105-190 \mathrm{~ms}$ amplitude $(\mu \mathrm{V})$ & $105-190 \mathrm{~ms}$ latency $(\mathrm{ms})$ \\
\hline Parieto-occipital & Deviant-minus-standard & $-0.67 \pm 0.15^{* * *}$ & $144 \pm 4.78$ \\
& Devinat-minus-equal probability control & $0.03 \pm 0.14$ & $149 \pm 10.50$ \\
& Deviant-minus-cascade control & $-0.09 \pm 0.09$ & $144 \pm 6.20$ \\
& & $118-148 \mathrm{~ms}$ amplitude $(\mu \mathrm{V})$ & $118-148 \mathrm{~ms}$ latency (ms) \\
Occipital & Deviant-minus-standard & $-0.43 \pm 0.14 * *$ & $134 \pm 5.03$ \\
& Deviant-minus-equal probability Control & $0.24 \pm 0.18$ & $124 \pm 6.14$ \\
& Deviant-minus-cascade control & $-0.04 \pm 0.10$ & $131 \pm 6.21$ \\
\hline
\end{tabular}




\begin{tabular}{|c|c|c|c|c|c|c|c|c|c|c|}
\hline & 6 & 9 & 12 & 15 & 18 & 21 & 24 & 27 & 30 & 33 \\
\hline & 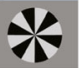 & 米 & 涉 & 症 & 政 & 諘监 & 諘/ & 畨 & 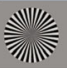 & 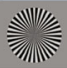 \\
\hline Oddball V6 & 96 & - & 864 & - & - & - & - & - & - & - \\
\hline Oddball V12 & 864 & - & 96 & - & - & - & - & - & - & - \\
\hline Equal Probability Control & 96 & 96 & 96 & 96 & 96 & 96 & 96 & 96 & 96 & 96 \\
\hline Modified Control V6 & 96 & 192 & 192 & 192 & 192 & - & - & - & - & - \\
\hline Modified Control V12 & - & - & 96 & 192 & 192 & 192 & 192 & - & - & - \\
\hline
\end{tabular}

Fig. 4 Stimuli and their probabilities of Experiment 2 (times/experiment) in the oddball and control sequences

rereferenced to average activity. The ground electrode was attached to the forehead. A horizontal EOG was recorded with a bipolar configuration between electrodes that were positioned lateral to the outer canthi of the two eyes. Vertical eye movements were monitored with a bipolar montage between electrodes that were placed above and below the right eye. The impedance of the electrodes was maintained below $10 \mathrm{k} \Omega$.

EEG signals were filtered off-line $(0.1-30 \mathrm{~Hz}, 24 \mathrm{~dB})$, and epochs of $500 \mathrm{~ms}$, starting from $100 \mathrm{~ms}$ before the stimulus onset, were averaged separately for the standards and deviants and for the control stimuli. Trials with an amplitude change that exceeded $+-100 \mathrm{uV}$ on any channel were rejected from further analysis.

Only the responses from the standard preceding a deviant were included in the standard-related ERPs. To identify the deviant-related effects, three types of grand-averaged differences were calculated: deviant-minus-standard, deviantminus-equal probability control, and deviant-minus-modified control. The difference in these comparisons cannot be explained by stimulus-specific features because the standard, deviant, and control stimuli had the same physical attributes.

The difference potentials were formed as follows:

V6 OddDiff $=$ V6 deviant-minus-V6 standard

$\mathrm{V} 12$ OddDiff $=$ V12 deviant-minus-V12 standard

V6 EQControlDiff = V6 deviant-minus-V6 E-control

V12 EQControlDiff $=$ V12 deviant-minus-V12 E-control

V6 MControlDiff = V6 deviant-minus-V6 M-control

V12 MControlDiff = V12 deviant-minus-V12 M-control

Similar to Experiment 1, we expected the emergence of vMMN at the posterior electrode sites. We defined an electrode matrix, where the OddV6Diff and OddV12Diff differed $(p<.01)$ from zero in at least 15 consecutive significant data points $(30 \mathrm{~ms})$, based on the results of a point-by-point t-test. A $2 \times 3$ matrix of channels $(\mathrm{PO} 3, \mathrm{POz}, \mathrm{PO} 4, \mathrm{O} 1, \mathrm{Oz}, \mathrm{O} 2)$ met this criterion, and it consisted of two rows (anterior, posterior) and three columns (left, middle, right). No laterality-related effect that was associated with the conditions was present, and thus, for the sake of easier traceability, we formed two regions of interest similar to Experiment 1: parieto-occipital (PO4, POz, PO3) and occipital $(\mathrm{O} 2, \mathrm{Oz}, \mathrm{O} 1)$.

To assess the effects of the conditions, the mean amplitude and peak latencies of the previously defined sections were measured. ANOVAs on the mean amplitudes and peak latencies were conducted, with the factors visual features (V6, V12), difference (OddDiff, EQControlDiff, MControlDiff), and ROI (parieto-occipital, occipital) on the difference potentials of the average amplitude values of the predefined ranges and on the peak latency values. The peak latencies were measured at the maxima of the differences.

The peak latencies and the scalp distributions of the exogenous components and the difference potentials were compared at the same ROIs. The effect size was characterized as partial eta-squared $\left(\eta_{\mathrm{p}}{ }^{2}\right)$. Where appropriate, post hoc analyses were calculated by the Tukey HSD test. Mauchly's test of sphericity was conducted when there were more than two conditions in the $F$ test. Surface distributions were compared under the method of the vector-scaled amplitude values (McCarthy \& Wood, 1985).

Considering that nose reference was used in the original study (Maekawa et al., 2005), nose-referenced data were also analyzed. Since there was no relevant difference between the nose and the averaged referenced data, only results computed from the average referenced data are presented.

\section{sLORETA analysis}

For defining the cortical location of the vMMN identified at the scalp level, we applied a distributed source localization technique. The source signal of the average ERP time series was reconstructed on the cortical surface by applying the sLORETA inverse solution (Pascual-Marqui, 2002). The sLORETA gives a solution for the EEG inverse problem by applying a weighted minimum norm estimation with spatial smoothing and standardization of the current density map. The forward model was generated on a realistic BEM head model (Gramfort, Papadopoulo, Olivi, \& Clerc, 2011) by applying a template MRI (ICBM152; $1 \mathrm{~mm}^{3}$ voxel resolution) with template electrode positions. The reconstructed dipoles $(\mathrm{pA} / \mathrm{m})$ were determined for every 15,001 sources in three orthogonal directions (unconstrained solution). The difference potentials were calculated for every voxel on source level between the same conditions as described previously on scalp level. For every voxel, the different potentials were averaged to 10 equal, $35-\mathrm{ms}$-long interval from zero to $350 \mathrm{~ms}$. Similarly to the scalp level, one-sample $t$ tests were applied on the difference potentials. Two conditions were reported as 
significant, if at least five voxels exceeded the Bonferronicorrected alpha level. Bonferroni correction was applied to control the Type I error that resulted from multiple comparisons of the 15,001 voxels and 10 time intervals of the condition pairs. Brain regions for the corresponding significant activations were identified based on the parcelation scheme introduced by Klein and Jason (2012).

\section{Results}

\section{Behavioural results}

The participants kept the ball inside the circle $97.88 \%$ of the time $(S E M=1.96 \%)$. There was no performance difference among the conditions.

\section{Event-related potentials}

As Fig. 5 shows, standard, deviant and control stimuli elicited a positive (P1), a negative (N1) and a positive (P2) series of peaks. It is obvious from Fig. 5 that there is a robust difference in both the amplitude and latency between the ERP elicited by stimuli comprised of six (V6) and 12 vanes (V12).

Figure 6 shows the grand average difference potentials, and Table 2 shows the mean amplitude and the latency values of the differences.

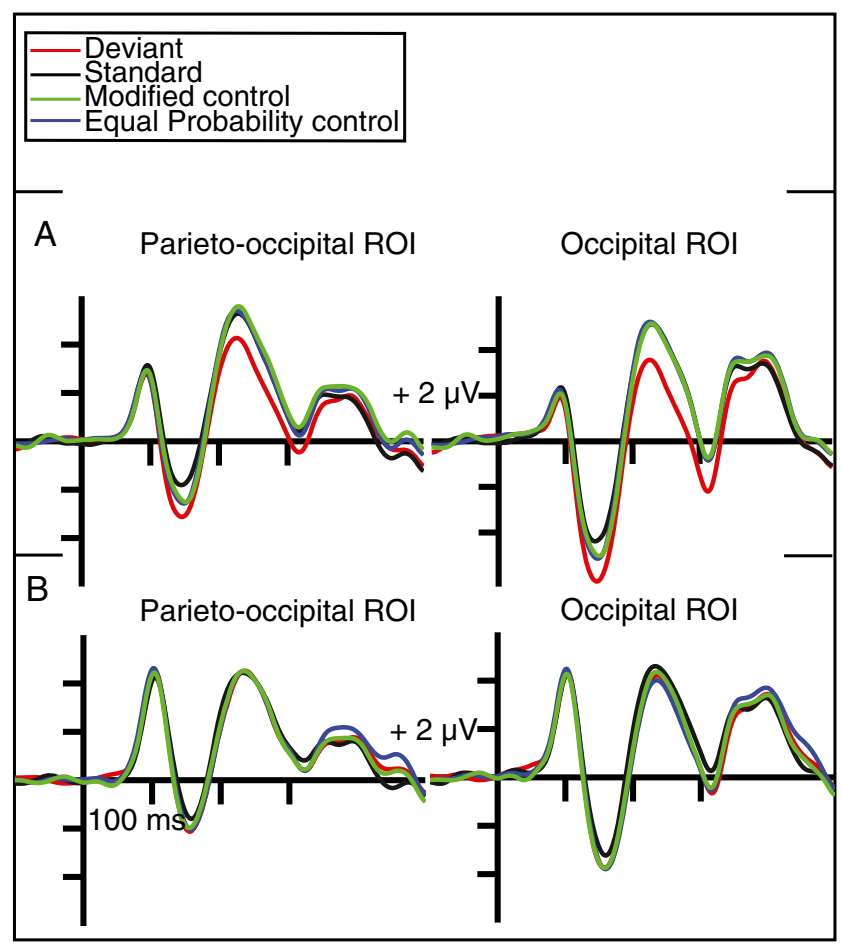

Fig. 5 Experiment 2: a Grand-averaged ERPs elicited by the V12 deviant, V12 standard, V12 equal probability control, and V12 modified control. b Grand-averaged ERPs elicited by the V6 deviant, V6 standard, V6 equal probability control, and V6 modified control. (Color figure online)
The point-by-point $t$ test conducted on the V12 OddDiff differed significantly from zero on the previously defined channel matrix in the 100-340 ms latency range. Based on the ERP wave characteristics, which is in agreement with Maekawa et al. (2005), we attempted to separate the difference into an early (100-200 ms) and a late (200-340 ms) latency range. However, the point-by-point $t$ test revealed restricted temporal and spatial extension for V6 OddDiff relative to V12 OddDiff; the deviant-minus-standard difference wave differed from zero in the 178-216 and in the 270-346 ms latency range at the occipital ROI. Investigating V6 and V12 separately was not planned a priori, but due to the large difference between them we have found it necessary to include a visual features factor into the design.

In the early time window (100-200 ms), the ANOVA on the amplitude values in the $100-200 \mathrm{~ms}$ revealed a significant main effect of visual features, $F(1,21)=5.75, p<.05, \eta_{\mathrm{p}}{ }^{2}=$ 0.21 ; V12 differences were more negative. The main effect of difference, $F(2,42)=12.02, p<.01, \eta_{\mathrm{p}}{ }^{2}=0.36$, indicated that the OddDiff values were more negative than the control differences. Tukey HSD post hoc analyses did not reveal a significant difference between EQControlDiff and MControlDiff. The Visual Features $\times$ ROI interaction, $F(1$, 21) $=5.14, p<.05, \eta_{\mathrm{p}}{ }^{2}=0.19$, revealed that V12 differences were more negative at the occipital site, while no such difference was specific for V6.

The ANOVA conducted on the latency values in the 100 $200 \mathrm{~ms}$ range with the same factors revealed a significant interaction of Visual Features $\times$ Difference, $F(2,42)=4.86$, $p<.05 ; \mathrm{V} 12$ OddDiff had a later peak than the V12 control differences.

The ANOVA on the amplitude values in the $200-340 \mathrm{~ms}$ also revealed a significant main effect of visual features, $F(1$, 21) $=11.92, p<.01, \eta_{\mathrm{p}}{ }^{2}=0.36$; the V12 differences were greater. The differences were greater at the occipital ROI, $F(1$, $21)=22.42, p<.01, \eta_{\mathrm{p}}{ }^{2}=0.51$. The Visual Features $\times$ Difference interaction, $F(2,42)=3.78, p<.05, \eta_{\mathrm{p}}{ }^{2}=0.15$, revealed that V12 OddDiff was significantly bigger than the control differences, while there was no difference in the mean amplitude values in response to V6.

\section{sLORETA results}

After the Bonferroni correction, significant difference of the difference potential from the baseline was identified in the V12 deviant-minus-V12 standard in the 105$245 \mathrm{~ms}$ range and in the V12 deviant-minus-V12 Mcontrol and in V12 deviant-minus-V12 E-control both in the 140-280 ms range. The sources were localized at the occipital areas; the lingual gyrus, cuneus and pericalcarine cortex showed the highest difference in the compared conditions (see Fig. 7 and Table 3). 


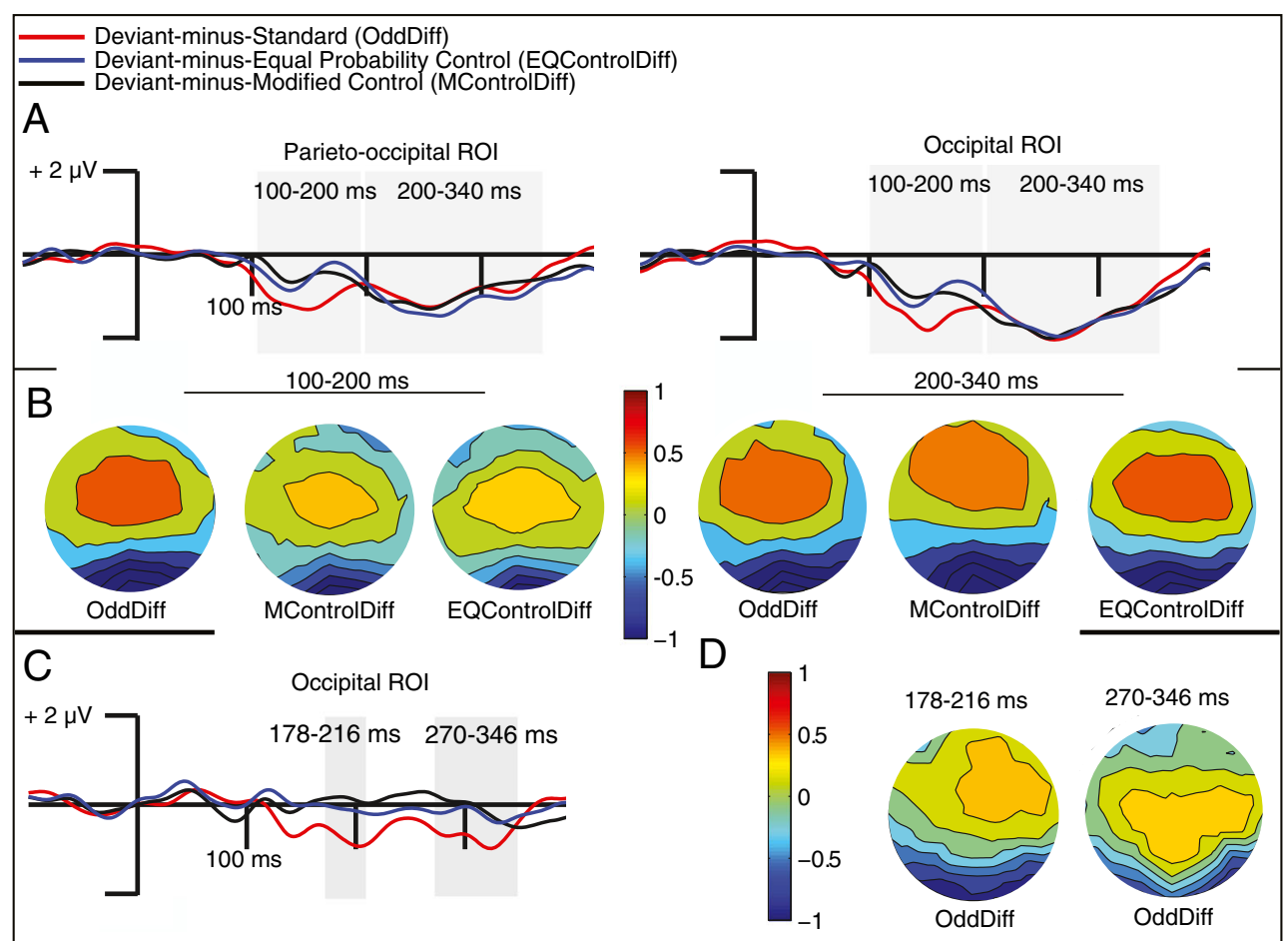

Fig. 6 Experiment 2: a Grand-averaged V12 deviant-minus-V12 standard, V12 deviant-minus-V12 modified control, and V12 deviantminus-V12 equal probability control difference waves. b Topographical maps of the V12 grand-averaged difference waves within the 100-200 ms and 200-350 ms time windows. c Grand-averaged V6 deviant-minus-V6

\section{Discussion}

For V12, there was a robust ERP difference in both the 100200 and 200-350 ms latency ranges. As the deviant-minuscontrol difference in the 100-200 ms range indicated, standard, V6 deviant-minus-V6 modified control, and V6 deviant-minusV6 equal probability control difference waves. $\mathbf{d}$ Topographical maps of the V6 grand-averaged difference waves within the 132-208 ms and 272$344 \mathrm{~ms}$ time windows. (Color figure online)

stimulus-specific adaptation explains a part of the observed difference but not all of it because both the deviant-minusequal probability control difference and the deviant-minusmodified control difference were different from zero, which indicates an adaptation-free gvMMN. In the later latency

Table 2 Experiment 2: Grand averages of peak latencies and mean epochs of the difference waves measured at parieto-occipital and occipital ROIs. Values are listed in mean \pm standard error. Difference wave significantly differ from zero. *.01 $>p<.05 ; * * p<.01$

\begin{tabular}{|c|c|c|c|c|c|}
\hline Region & Difference waves & $\begin{array}{l}\text { 100-200 ms amplitude } \\
(\mu \mathrm{V})\end{array}$ & $\begin{array}{l}\text { 100-200 ms latency } \\
(\mathrm{ms})\end{array}$ & $\begin{array}{l}\text { 200-340 ms amplitude } \\
(\mu \mathrm{V})\end{array}$ & $\begin{array}{l}\text { 200-340 ms latency } \\
\text { (ms) }\end{array}$ \\
\hline \multirow[t]{3}{*}{ Parieto-occipital } & $\begin{array}{l}\text { V12 deviant-minus-V12 } \\
\text { standard }\end{array}$ & $-1.01 \pm 0.26^{* *}$ & $150 \pm 6.13$ & $-0.92 \pm 0.26^{* *}$ & $291 \pm 10.02$ \\
\hline & $\begin{array}{l}\text { V12 deviant-minus-V12 } \\
\text { E-control }\end{array}$ & $-0.48 \pm 0.20 *$ & $158 \pm 9.24$ & $-0.91 \pm 0.23 * *$ & $263 \pm 10.68$ \\
\hline & $\begin{array}{l}\text { V12 deviant-minus-V12 } \\
\text { M-control }\end{array}$ & $-0.55 \pm 0.23 *$ & $152 \pm 7.19$ & $-1.05 \pm 0.20 * *$ & $274 \pm 8.54$ \\
\hline \multirow[t]{4}{*}{ Occipital } & $\begin{array}{l}\text { V12 deviant-minus-V12 } \\
\text { Standard }\end{array}$ & $-1.37 \pm 0.24 * *$ & $161 \pm 6.10$ & $-1.46 \pm 0.26^{* *}$ & $272 \pm 8.52$ \\
\hline & $\begin{array}{l}\text { V12 deviant-minus-V12 } \\
\text { E-control }\end{array}$ & $-0.82 \pm 0.21 * *$ & $162 \pm 7.93$ & $-1.49 \pm 0.26^{* *}$ & $269 \pm 10.56$ \\
\hline & $\begin{array}{l}\text { V12 deviant-minus-V12 } \\
\text { M-control }\end{array}$ & $-0.81 \pm 0.22 * *$ & $164 \pm 7.37$ & $-1.40 \pm 0.25 * *$ & $269 \pm 9.00$ \\
\hline & & $\begin{array}{l}\text { 178-216 ms } \\
\text { amplitude }(\mu \mathrm{V})\end{array}$ & $\begin{array}{l}\text { 178-216 ms } \\
\text { latency (ms) }\end{array}$ & $\begin{array}{l}270-346 \mathrm{~ms} \\
\text { amplitude }(\mu \mathrm{V})\end{array}$ & $\begin{array}{l}270-346 \mathrm{~ms} \\
\text { latency (ms) }\end{array}$ \\
\hline \multirow[t]{3}{*}{ Occipital } & V6 deviant-minus-V6 standard & $-0.84 \pm 0.22 * *$ & $191 \pm 6.64$ & $-0.77 \pm 0.24 * *$ & $314 \pm 6.63$ \\
\hline & V6 deviant-minus-V6 E-control & $0.10 \pm 0.21$ & $194 \pm 4.82$ & $0.06 \pm 0.28$ & $330 \pm 4.31$ \\
\hline & $\begin{array}{l}\text { V6 deviant-minus-V6 } \\
\text { M-control }\end{array}$ & $0.00 \pm 0.23$ & $192 \pm 5.01$ & $-0.09 \pm 0.24$ & $316 \pm 7.33$ \\
\hline
\end{tabular}




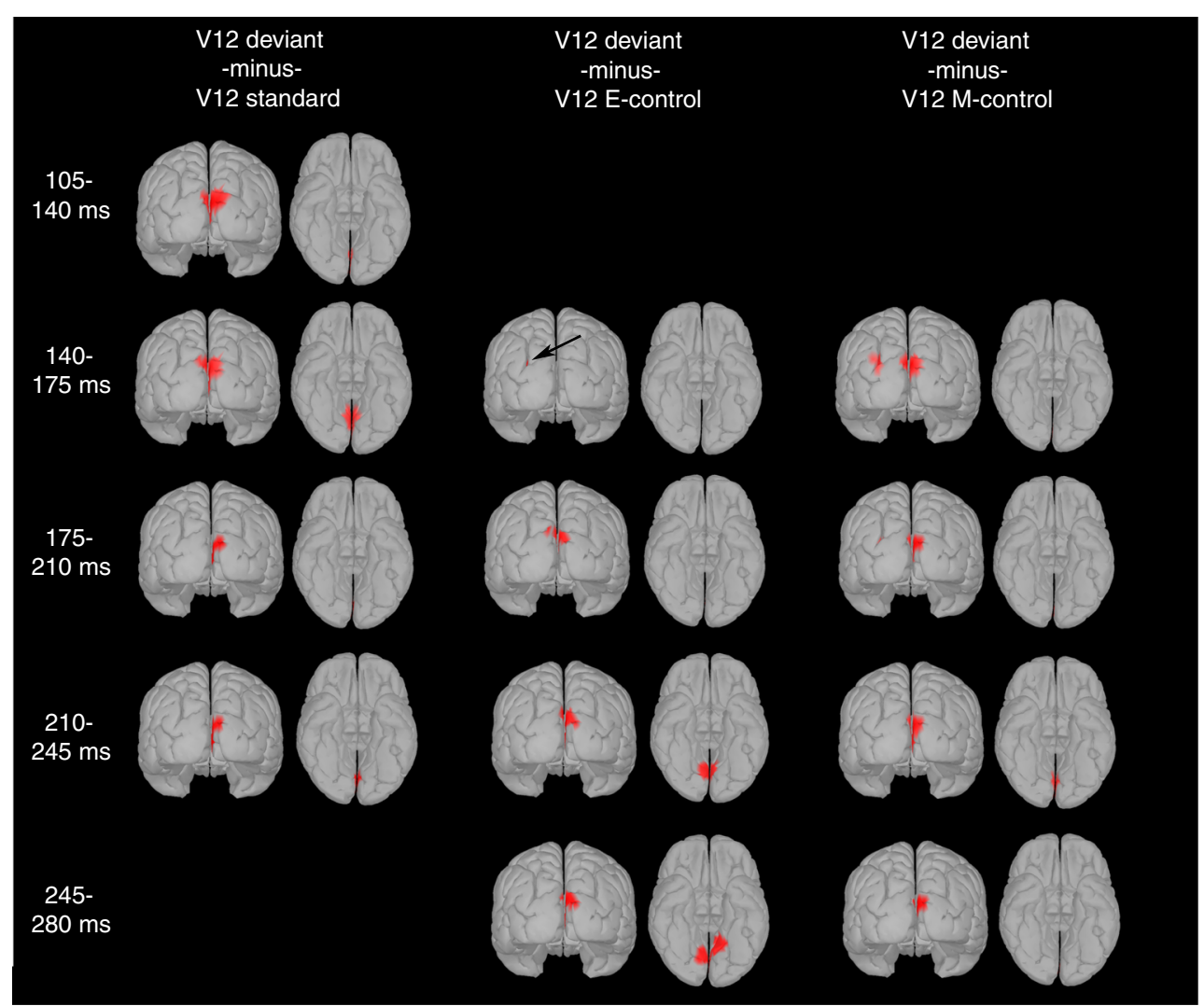

Fig. 7 Experiment 2: Source distribution of the significant differences between the difference potentials and baseline. Red colors indicating the significant voxels. For every voxel, the difference potentials were averaged to 10 equal, $35-\mathrm{ms}-$ long intervals from zero to $350 \mathrm{~ms}$.

range, the V12 deviant-minus-standard and the deviantminus-control differences were identical, and thus, the deviant effects cannot be attributed to adaptation processes. In contrast to the results on the V12 stimuli, for V6, the control differences abolished $\mathrm{vMMN}$ in both of the latency ranges. According to the prevailing explanation, this finding means that the deviant-minus-standard difference was due to adaptation. For an adaptation explanation of the asymmetry between the effects of the V12 and V6 patterns, sequential presentation of V12 adapts the neuronal population sensitive to V6 (in other words, representation of the more complex stimulus included the representation of the less complex stimulus). However, the continuous presentation of V6 did not adapt the cell population that is responsible for V12. The V6 deviant does not elicit gvMMN because it did not carry new information within the sensory system. As a low-level variant of this account, all of the edges that form V6 are present in V12. This finding means that the contrast border orientations of $\mathrm{V} 6$ are present in V12, but not vice versa. When considering the S6D12 oddball sequence, the orientations that form the V6 stimuli are presented 180 times $(100 \%)$, while the orientations that are specific to V12 are present only 18 times $(10 \%)$. However, in the S12D6 sequence, the orientations that form the V6 stimuli are presented 180 times again, while the V12-
Difference potentials of two conditions on a given interval reported as significant, if at least five voxels exceeded the Bonferroni corrected alpha level. (Color figure online)

specific orientations present 162 times (90\%). This finding could lead to the observed asymmetry because a less pronounced stimulus-specific adaptation effect is expected for the V12 deviant compared with the V6. The difference between the explanations is conceptual. The former explanation emphasizes higher order (also less precisely defined) features (i.e., complexity), while the latter explanation is based on elementary visual features. Overall, the V12-V6 asymmetry requires further study, especially in light of other vMMN asymmetries (Kecskés-Kovács et al., 2013b; Sulykos et al., 2015).

\section{Experiment 3}

In Experiment 3, we investigated a possible source of the different deviancy effects of the windmill patterns with six (V6) and 12 (V12) vanes. We presented two additional oddball sequences that had stimuli with four (V4) and six (V6) windmill patterns (i.e., the S4D6 and S6D4 sequences). In these sequences, the probabilities of having spatially matching edge orientations were balanced. In the S4D6 sequence, the probability of matching edge orientations within the standard was 0.95 and 0.4 for the deviant, while in the S6D4 sequence, 

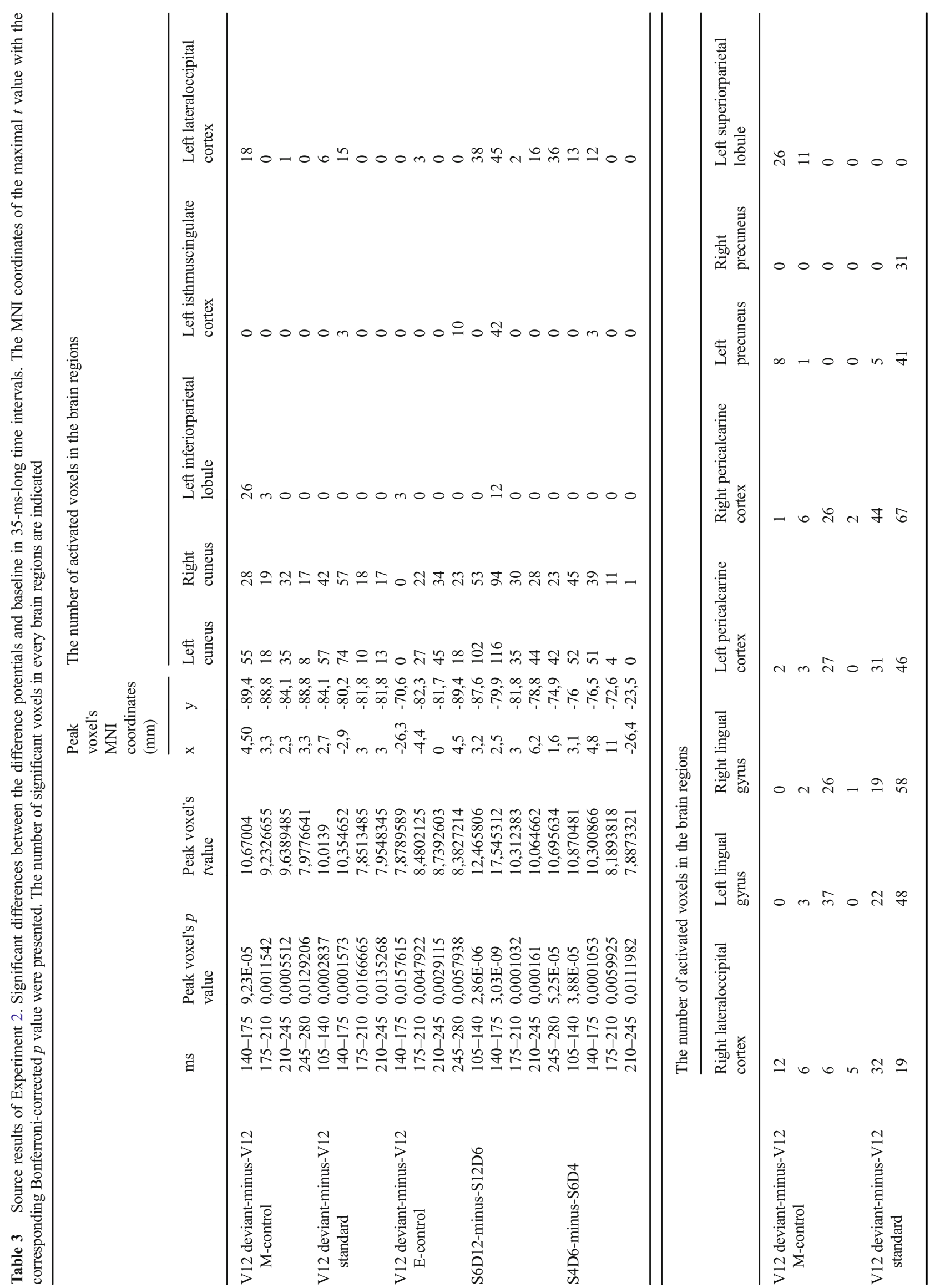


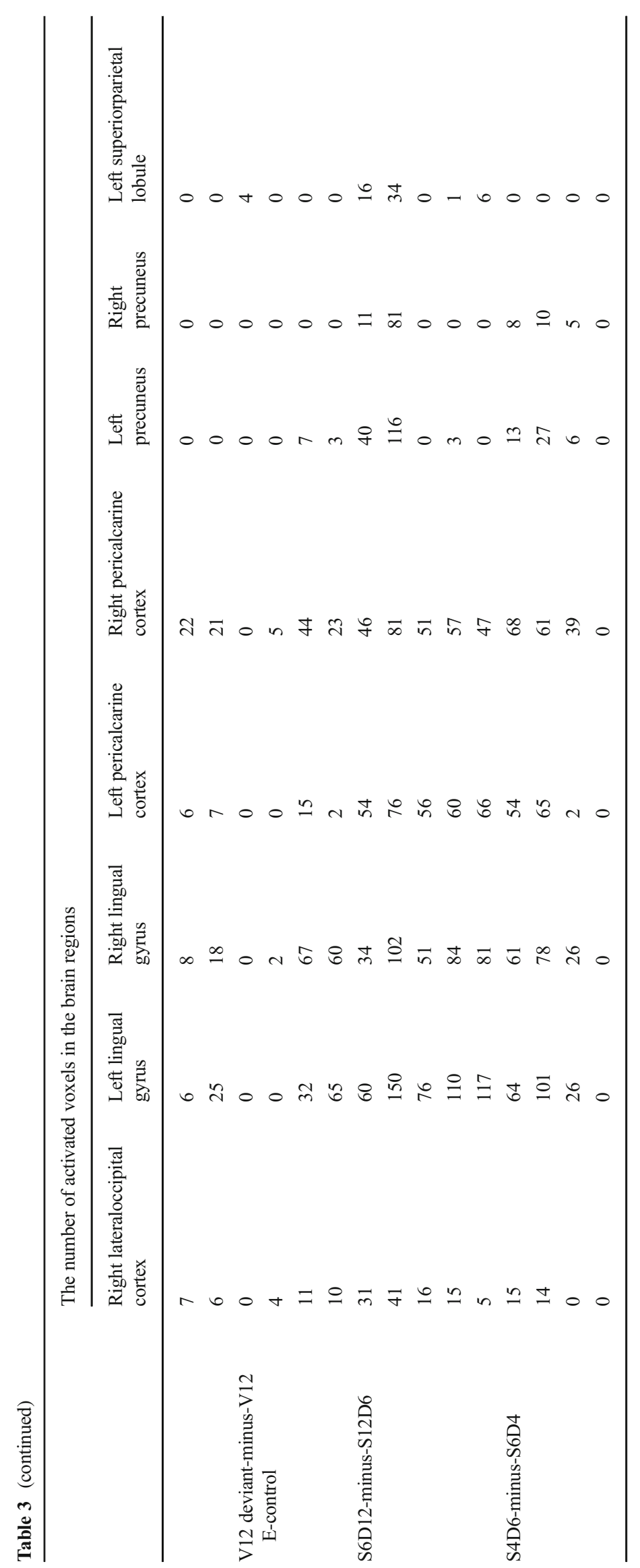




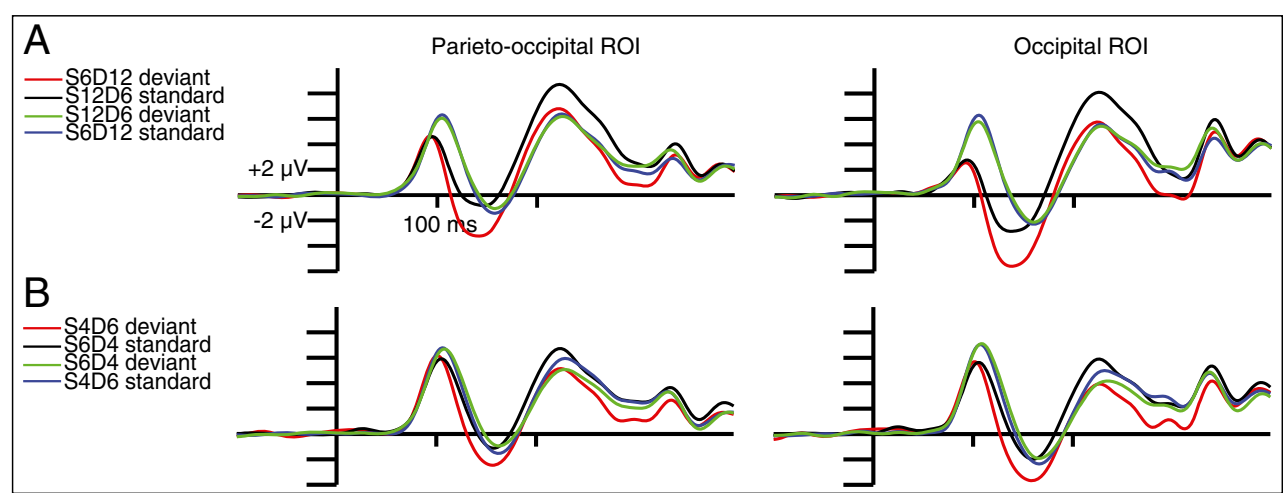

Fig. 8 Experiment 3: a Grand-averaged ERPs elicited by the S6D12 deviant, S12D6 standard, S12D6 deviant, and S6D12 standard. b Grand-averaged ERPs elicited by the S4D6 deviant, S6D4 standard, S6D4 deviant, and S4D6 standard. (Color figure online)

it was 0.93 for the standard and 0.55 for the deviant. If the same asymmetry occurs between the V4 and V6 differences than in the case of the V6 and V12, the result favors the complexity explanation over the orientation specificity (lowlevel) account. In an attempt to replicate the results of Experiment 2, oddball sequences with V6 and V12 stimuli were also delivered.

\section{Method}

\section{Participants}

Twenty-five volunteers participated in the study for monetary compensation or for course credit. They had no ophthalmologic or neurological abnormalities. Written consent was obtained from all of the participants prior to the experimental procedure. The study was conducted in accordance with the Declaration of Helsinki and approved by the Joint Committee of Ethics of the Psychology Institute in Hungary. Two out of 25 participants were omitted from the data analyses due to the low signal-to-noise ratio, and thus, we report the data from 23 participants ( 18 women; mean age $=21.37 ; S D=1.83$ years).

\section{Stimuli and experimental design}

Four different conditions were used; S6D12, S12D6, S4D6, and S6D4.

\section{Stimuli and procedure}

All of the aspects of the stimulations in Experiment 3 were identical to those of the stimuli that we applied in Experiment 2.

\section{Recording and measuring the electrical brain activity}

The parameters of the EEG-recording, the processing of the EEG-signal, and the statistical analyses of the behavioral data and the ERP data were identical to Experiment 2.

The differences were formed as follows:

$$
\begin{aligned}
& \text { S6D12Diff = S6D12-minus-S12D6 } \\
& \text { S12D6Diff = S12D6-minus-S6D12 } \\
& \text { S4D6Diff = S4D6-minus-S6D4 } \\
& \text { S6D4Diff = S6D4-minus-S4D6 }
\end{aligned}
$$

The same $2 \times 3$ matrix of channels (PO3, POz, PO4, O1, $\mathrm{Oz}, \mathrm{O} 2$ ) met the criteria that we set in Experiment 2. The occipital and parieto-occipital channels did not differ from each other, and thus, for easier traceability, two regions of interest were formed: occipital and parieto-occipital.

The point-by-point $t$ test ( $p=.01$ ) on the S6D12Diff differed significantly from zero in the $102-340 \mathrm{~ms}$ and in the 110-340 latency ranges on the S4D6Diff. Similar to in Experiment 2, we divided the difference potentials into an early (100-200 ms) and a late (200-340 ms) latency range,

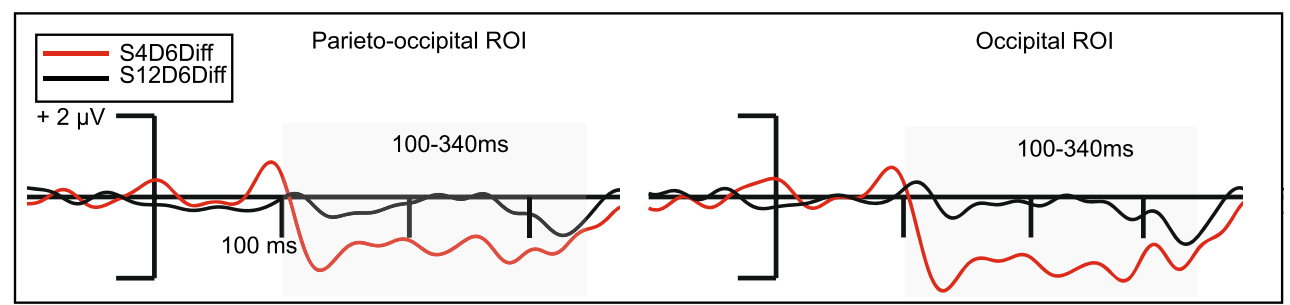

Fig. 9 Experiment 3: Grand-averaged V6 deviant-minus-V4 standard and V6 deviant-minus-V12 standard difference waves. (Color figure online) 
Table 4 Experiment 3: Grand averages of peak latencies and mean epochs of the difference waves measured at parieto-occipital and occipital ROIs.Values are listed in mean \pm standard error. Difference wave significantly differ from zero. $* .01>p<.05 ; * *<.01$

\begin{tabular}{|c|c|c|c|c|c|}
\hline Region & Difference waves & $\begin{array}{l}\text { 100-200 ms } \\
\text { amplitude }(\mu \mathrm{V})\end{array}$ & $\begin{array}{l}100-200 \mathrm{~ms} \\
\text { latency (ms) }\end{array}$ & $\begin{array}{l}200-340 \mathrm{~ms} \\
\text { amplitude }(\mu \mathrm{V})\end{array}$ & $\begin{array}{l}200-340 \mathrm{~ms} \\
\text { latency }(\mathrm{ms})\end{array}$ \\
\hline \multirow[t]{2}{*}{ Parieto-occipital } & V12 deviant-minus-V12 standard & $-1.82 \pm 0.28 * *$ & $149 \pm 5.09$ & $-1.73 \pm 0.42 * *$ & $268 \pm 6.97$ \\
\hline & $\begin{array}{l}\text { V6 deviant-minus-V6 standard } \\
\text { (in V4 sequence) }\end{array}$ & $-1.25 \pm 0.21^{* *}$ & $156 \pm 6.94$ & $-1.37 \pm 0.26^{* *}$ & $270 \pm 10.59$ \\
\hline \multirow[t]{2}{*}{ Occipital } & V12 deviant-minus-V12 standard & $-2.06 \pm 0.30^{* *}$ & $158 \pm 6.93$ & $-2.04 \pm 0.42 * *$ & $268 \pm 7.24$ \\
\hline & $\begin{array}{l}\text { V6 deviant-minus-V6 standard } \\
\text { (in V4 sequence) }\end{array}$ & $-1.58 \pm 0.21 * *$ & $150 \pm 6.55$ & $-1.69 \pm 0.28 * *$ & $275 \pm 9.77$ \\
\hline
\end{tabular}

based on the characteristics of the subcomponents of S6D12Diff (the difference wave components were not evident in the case of S4D6Diff). There were no sections in the S6D4Diff and S12D6Diff in which they differed from zero for at least 15 consecutive data points $(30 \mathrm{~ms})$.

To assess the effects of the conditions, two-way ANOVAs were conducted on the difference waves' amplitude values and latency values with the factors of differences (S6D12, S4D6) and ROI (occipital, parieto-occipital).

\section{sLORETA analysis}

The S4D6 versus S6D4 conditions were statistically evaluated at the source level, using methodology that was identical to that used in Experiment 2.

\section{Results}

\section{Behavioral results}

The participants kept the ball inside the circle $97.80 \%$ of the time $(S E M=0.8 \%)$. There was no difference in their performances between the conditions.

\section{Event-related potentials}

Similar to in Experiment 2, a positive (P1)-negative (N1)positive (P2) deflection was elicited by both the standard and deviant stimuli (see Fig. 8).

The ANOVA conducted in the 100-200 and 200-340 ms ranges on the amplitude values and peak latency values of the S6D12Diff and S4D6Diff revealed no difference. Figure 9 shows the difference waves of the V6 stimuli in the sequence in which V4 (S4D6Diff) and V12 (S12D6Diff) were the standard stimuli. Table 4 shows the amplitude and latency values of the differences.

\section{sLORETA results}

After the Bonferroni correction, significant difference of the difference potential from the baseline was identified in the S6D12-minus-S12D6 in the 105-280 ms range and in the S4D6-minus-S6D4 in the 105-245 ms range. Similarly to Experiment 2, the sources were localized to the occipital areas (see Fig. 10 and Table 3 for a detailed description).

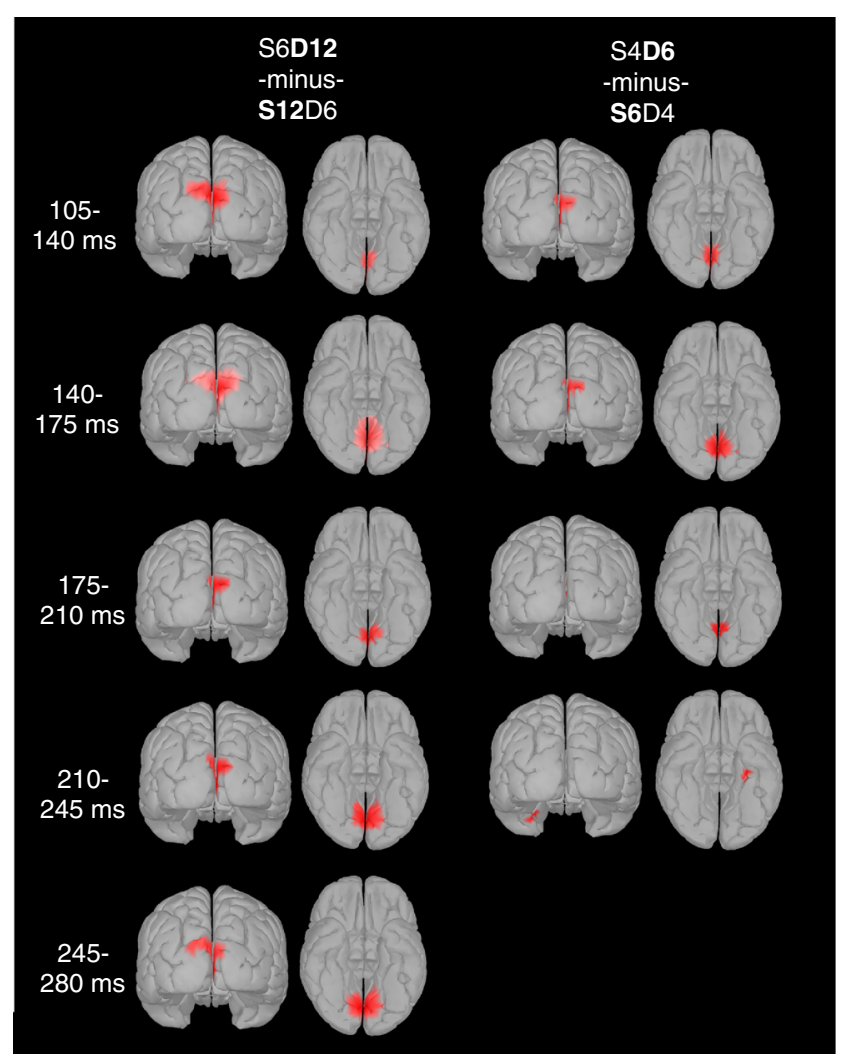

Fig. 10 Experiment 3: Source distribution of the significant differences between the difference potentials and baseline. Red colors indicating the significant voxels. For every voxel, the difference potentials were averaged to 10 equal, $35-\mathrm{ms}$-long intervals from zero to $350 \mathrm{~ms}$. Difference potentials of two conditions on a given interval reported as significant, if at least five voxels exceeded the Bonferroni-corrected alpha level. (Color figure online) 


\section{Discussion}

A robust negative deflection was observable in the S6D12Diff and S4D6Diff waves in the 100-200 and 200-340 ms latency ranges. However, no negative deflection was observable in the S12D6Diff and S6D4Diff waves. These results support the notion that the observed asymmetry between the V6 and V12 difference waves is due to the complexity differences rather than the overlapping edges in the sequentially presented stimuli.

\section{Reliability of Experiment 2 and 3}

To test the reliability of Experiment 2 and 3, independentsamples $t$ tests were conducted on the amplitude values of the cognate difference waves measured in Experiment 2 and 3 (i.e., V6 OddDiff-S12D6Diff and V12 OddDiff-S6D12Diff).

There was no significant difference between the amplitude values of V12 OddDiff and S6D12Diff in the early (100-200 $\mathrm{ms})$ and in the late (200-340 ms) range, either at the occipital or the parieto-occipital ROIs.

There was significant difference between the amplitude values of V6 OddDiff $(M=-0.86, S D=1.14)$ and S12D6Diff $(M=-0.08, S D=1.21)$ in the early $(178-216$ $\mathrm{ms}$ ) range at the occipital ROI; $t(43)=2.19, p<.05$. No such difference was observable at the parieto-occipital ROI. There was no significant difference in the late (270-346 ms) differences between the two recording sessions.

\section{General discussion}

In previous visual oddball studies, the deviant-minus-standard difference wave has been found to be negative at approximately 100-350 ms (e.g., Czigler et al., 2006; Kimura et al., 2009; Takács et al., 2013; however, see Czigler \& Sulykos, 2010). This negativity is often labeled as vMMN regardless of its size, extension or occurrence in time. Despite the many studies that have been conducted in the field of vMMN research, it is still an open issue as to whether this negativity is a correlate of a memory comparison/prediction process (Czigler, 2007) or a consequence of a putatively more simple process (i.e., refractoriness/habituation/repetition suppression/ stimulus specific adaptation). A possible explanation for the contradictory experimental results is that one uniform underlying mechanism that would generate vMMN does not exist and that instead there are separate mechanisms, depending on the circumstances/stimulation. Is it computationally effective to build a memory trace of certain orientations, when the same information is already present in the adaptational states of the efferent neurons? However, higher level regularities probably cannot be coded on the level of sensory neuronal adaptation, and thus, the presence of a memory comparison process is justifiable.

In our study, we demonstrated that for simple features, such as orientation, SSA is a sufficient method for coding the regularities; the results of Experiment 1 can be fully explained by SSA. In the 105-190 ms range at parieto-occipital ROI and in the 118-148 ms range at the occipital ROI, the ERPs to the deviant and the control stimuli did not differ from each other, but they both elicited more negative responses than the standard, which supports the notion that the MMN is a modulation of the exogenous activity. Because the latency of the difference potential was similar to the latency of N1, the results fit the claim of May and Tiitinen (2010) and Kimura et al. (2009). This result is in line with the findings in a study by Kenemans et al. (2003). This group presented a "lonely deviant" sequence (i.e., a sequence that consists of only rarely presented identical stimuli). The ERPs that are elicited by the lonely deviant and by the oddball deviant did not differ from each other in their latency and scalp distribution, which supports the notion that $\mathrm{vMMN}$ is better explained by stimulus rarity than by a mismatch process (Kenemans et al., 2003).

However, the results of Experiments 2 and 3 were markedly different and cannot be explained solely with SSA. They fit partly with the results of Maekawa et al. (2005) and partly support the findings of Kimura et al. (2009), Kimura et al. (2015), and Czigler et al. (2002) (i.e., in terms of the concept of memorycomparison-based change detection). In concordance with Kimura et al. (2009), in the early range of the deviant-minusstandard difference, SSA was present, as the deviant-minuscontrol did not differ from zero in that range. However, contrary to Kimura's findings, in Experiment 2, gvMMN was obtainable too in the early, 100-200 ms range. The later range was absent of SSA because the oddball and the control differences were almost identical. It is important to note here, that the interstimulus interval (800 ms) used in our experiment is considerably larger, than in most vMMN studies, which might contribute to some of the differences observed in our results.

Traditionally in vMMN studies, the equal probability control is used to separate the memory-comparison and adaptation-based effects (i.e., Kimura et al., 2009; Kimura et al., 2015). Here, we tested two additional paradigms, which were adapted from the auditory field. The difference waves that were formed from the various controls were almost identical. Considering this finding, we support the usage of equal probability control, which leads to better comparability among the vMMN studies.

The experimental protocol enabled us to investigate separately the neural generators of the deviant-related activity, which reflects partly SSA and gvMMN. The present results with respect to the visual areas are highly consistent with previous findings: Kimura et al. (2010) identified the source of MMN to the right cuneus and to the frontal lobe; Urakawa, Inui, Yamashiro and Kakigi (2010) indicated that the 
precuneus, while Susac et al. (2013) found that the middle occipital gyrus and cuneus is involved in the deviant-related activity. These results support the notion that preattentive change detection is a relatively low level, modality-specific process in the visual cortex (Susac et al., 2013).

For the asymmetry of the V6 and V12 deviancy effects, the complexity-related explanation was supported by the results of Experiment 3 (an asymmetry between the V4 and V6 effects). According to this account, the V4 deviant within the sequence of $\mathrm{V} 6$ does not elicit vMMN because it did not carry new information within the sensory system.

This possibility is in line with the predictive coding account of mismatch responses (in both the auditory and visual modality), which leads to the claim that redundancy reduction is an essential feature of efficient coding. Direct representation of the raw images appears to be inefficient, and therefore, a possible role of a stimulus processing stage is to recode the sensory input into an efficient form. According to the predictive coding view, neuronal networks learn the statistical regularities of the world and reduce redundancy by transmitting only the unpredicted portions of an incoming sensory signal (Huang \& Rao, 2011). The suggested mechanism relies on hierarchically organized neural systems in which top-down connections carry predictions from higher level to lower level areas, and bottom-up connections ensure the updating of the predictive models. As Garrido et al. (2009) highlight, the model adjustment hypothesis, which considers the $\mathrm{MMN} / \mathrm{vMMN}$ to be an error signal that is elicited by a deviation from a learned regularity, is completely consistent with the predictive coding framework (Kimura, 2012; Näätänen \& Winkler, 1999; Stefanics et al., 2014; Winkler \& Czigler, 2012; Winkler et al., 1996). In other words, MMN/vMMN is a correlate of a bottom-up signal that is responsible for the updating of the predictive model, and in this way, it minimizes the computational costs in higher areas (Garrido et al., 2009).

Based on our results, the conclusion of Kimura et al. (2009) - according to which the deviant-minus-standard difference wave consists of two subsequent posterior negativities reflecting adaptation effect and memory-comparison-based change detection effect-is supplemented with the observation, that the proportion of adaptation and memory effect in vMMN are not constant between experiments. The different level of adaptation among experimental settings might also contribute to the reported feature-related vMMN (Sulykos \& Czigler, 2011; Susac et al., 2013) and is against the assumption, that vMMN is completely attributable to adaptation, and strengthen the notion that adaptation might have a functional role in the process of automatic change detection and not just a byproduct of repetition.

\section{Conclusions}

In Experiments 1 and 2, we obtained highly different ERP effects, which suggests that as a function of the level of the deviance, different underlying mechanisms are responsible for the production of the observed negativity in the deviantminus-standard difference wave. Additionally, the results of Experiments 2 and 3 highlight that the presented stimuli in the oddball sequence interact and that the complexity difference between the infrequent and frequent stimuli has considerable influence on the deviant-related response.

Acknowledgments This study was supported by the Hungarian Research Fund (OTKA 104462). We thank Zsuzsa d'Albini and Zsuzsa Kovács for their technical help.

There are no conflicts of interest.

\section{References}

Amando, C., \& Kovács, G. (2016). Does surprise enhancement or repetition suppression explain visual mismatch negativity? European Journal of Neuroscience, 43, 1590-1600.

Astikainen, P., Cong, F., Ristaniemi, T., \& Hietanen, J. (2013). Eventrelated potentials to unattended changes in facial expressions: Detection of regularity violations or encoding of emotions? Frontiers in Human Neuroscience, 7, 557.

Astikainen, P., \& Hietanen, J. K. (2009). Event-related potentials to taskirrelevant changes in facial expressions. Behavioral and Brain Functions, 5, 30.

Astikainen, P., Lillstrang, E., \& Ruusuvirta, T. (2008). Visual mismatch negativity for changes in orientation - A sensory memorydependent response. European Journal of Neuroscience, 28, 2319-2324.

Astikainen, P., Ruusuvirta, T., Wikgren, J., \& Korhonen, T. (2004). The human brain processes visual changes that are not cued by attended auditory stimulation. Neuroscience Letters, 368(2), 231-234.

Clifford, C. W. G. (2002). Perceptual adaptation: Motion parallels orientation. Trends in Cognitive Sciences, 6, 136-143.

Csukly, G., Stefanics, G., Komlósi, S., Czigler, I., \& Czombor, P. (2013). Emotion-related visual mismatch responses in schizophrenia: Impairments and correlations with emotion recognition. PLoS ONE, 8(10), e75444. doi:10.1371/journal.pone.0075444

Czigler, I. (2007). Visual mismatch negativity_-Violation of nonattended environmental regularities. Journal of Psychophysiology, 21, 224 230.

Czigler, I., Balázs, L., \& Winkler, I. (2002). Memory-based detection of task-irrelevant visual changes. Psychophysiology, 39, 869-873.

Czigler, I., \& Sulykos, I. (2010). Visual mismatch negativity to irrelevant changes is sensitive to task-relevant changes. Neuropsychologia, 48(5), 1277-1282.

Czigler, I., Weisz, J., \& Winkler, I. (2006). ERPs and deviance detection: Visual mismatch negativity to repeated visual stimuli. Neuroscience Letters, 401(1/2), 178-182.

Friston, K. (2003). Learning and inference in the brain. Neural Networks, 16, 1325-1352.

Friston, K. (2005). A theory of cortical responses. Philosophical Transactions of the Royal Society of London. Series B, Biological Sciences, 360, 815-836.

Garrido, M., Kilner, J., Stephan, K., \& Friston, K. (2009). The mismatch negativity: A review of underlying mechanisms. Clinical Neurophysiology, 120, 453-463.

Gibson, J. (1937). Adaptation with negative after-effect. Psychological Review, 44(3), 222-244. 
Gramfort, A., Papadopoulo, T., Olivi, E., \& Clerc, M. (2011). Forward field computation with Open MEEG. Computational Intelligence and Neuroscience, 2011. doi:10.1155/2011/923703

Heslenfeld, D. J. (2002). Visual mismatch negativity. In J. Polich (Ed.), Detection of change: Event-related potential and fMRI findings. Dordrecht, Netherlands: Kluwer.

Huang, Y., \& Rao, R. (2011). Predictive coding. Wiley Interdisciplinary Reviews: Cognitive Science, 2, 580-593.

Jacobsen, T., \& Schroger, E. (2001). Is there pre-attentive memory-based comparison of pitch? Psychophysiology, 38, 723-727.

Jacobsen, T., Schröger, E., Horenkamp, T., \& Winkler, I. (2003). Mismatch negativity to pitch change: Varied stimulus proportions in controlling effects of neural refractoriness on human auditory event-related brain potentials. Neuroscience Letters, 344(2), 79-82.

Kecskés-Kovács, K., Sulykos, I., \& Czigler, I. (2013a). Is it a face of a woman or a man? Visual mismatch negativity is sensitive to gender category. Frontiers in Human Neuroscience, 7, 532.

Kecskés-Kovács, K., Sulykos, I., \& Czigler, I. (2013b). Visual mismatch negativity is sensitive to symmetry as a perceptual category. The European Journal of Neuroscience, 37(4), 662-667.

Kenemans, J. L., Jong, T. G., \& Verbaten, M. N. (2003). Detection of visual change: Mismatch or rareness? NeuroReport, 14(9), 12391242.

Kimura, M. (2012). Visual mismatch negativity and unintentional temporal-context-based prediction in vision. International Journal of Psychophysiology, 83, 144-155.

Kimura, M., Katayama, J., Ohira, H., \& Schröger, E. (2009). Visual mismatch negativity: New evidence from the equiprobable paradigm. Psychophysiology, 46, 402-409.

Kimura, M., Ohira, H., \& Schröger, E. (2010). Localizing sensory and cognitive systems for pre-attentive visual deviance detection: An sLORETA analysis of the data of Kimura et al. (2009). Neuroscience Letters, 485, 198-203.

Kimura, M., \& Takeda, J. (2013). Task difficulty affects the predictive process indexed by visual mismatch negativity. Frontiers in Human Neuroscience, 7. doi:10.3389/fnhum.2013.00267

Kimura, M., \& Takeda, Y. (2015). Automatic prediction regarding the next state of a visual object: electrophysiological indicators of prediction match and mismatch. Brain Research, 1626, 31-44

Klein, A., \& Jason, T. (2012). 101 labeled brain images and a consistent human cortical labeling protocol. Frontiers in Neuroscience, 6(2012), 171.

Krekelberg, B., Boyton, G., \& Wezel, R. (2006). Adaptation: From single cells to BOLD signals. Trends in Neurosciences, 29(5), 250-256.

Li, X., Lu, Y., Sun, G., Gao, L., \& Zhao, L. (2012). Visual mismatch negativity elicited by facial expressions: New evidence from the equiprobable paradigm. Behavioral and Brain Functions, 8(1), 7. doi:10.1186/1744-9081-8-7

Maekawa, T., Goto, Y., Kinukawa, N., Taniwaki, T., Kanba, S., \& Tobimatsu, S. (2005). Functional characterization of mismatch negativity to a visual stimulus. Clinical Neurophysiology, 116, 23922402.

May, P. J. C., \& Tiitinen, H. (2010). Mismatch negativity (MMN), the deviance-elicited auditory deflection, explained. Psychophysiology, 47(1), 66-122.

McCarthy, G., \& Wood, C. C. (1985). Scalp distributions of event-related potentials: An ambiguity associated with analysis of variance models. Electroencephalography and Clinical Neurophysiology, 62(3), 203-208. doi:10.1016/0168-5597(85)90015-2

Näätänen, R., \& Winkler, I. (1999). The concept of auditory stimulus representation in cognitive neuroscience. Psychological Bulletin, $125(6), 826-859$.
O'Shea, R. (2015). Refractoriness about adaptation. Frontiers in Human Neuroscience, 9(38), 1-3.

Pascual-Marqui, R. D. (2002). Standardized low-resolution brain electromagnetic tomography (sLORETA): Technical details. Methods and Findings in Experimental and Clinical Pharmacology, 24(Suppl. D), 5-12.

Pazo-Alvarez, P., Amenedo, E., \& Cadaveira, F. (2004). Automatic detection of motion direction changes in the human brain. The European Journal of Neuroscience, 19(7), 1978-1986.

Rensink, A. R. (2002). Change detection. Annual Review of Psychology, 53, 245-277.

Ruhnau, P., Herrmann, B., \& Schröger, E. (2012). Finding the right control: The mismatch negativity under investigation. Clinical Neurophysiology, 123(3), 507-512.

Sawamura, H., Orban, G. A., \& Vogels, R. (2006). Selectivity of neuronal adaptation does not match response selectivity: A single-cell study of the FMRI adaptation paradigm. Neuron, 49, 307-318.

Schröger, E., \& Wolff, C. (1996). Mismatch response of the human brain to changes in sound location. NeuroReport, 7, 3005-3008.

Simons, D. J., \& Levin, D. T. (1997). Change blindness. Trends in Cognitive Science, 1, 261-267.

Stefanics, G., Csukly, G., Komlósi, S., Czobor, P., \& Czigler, I. (2012). Processing of unattended facial emotions: A visual mismatch negativity study. NeuroImage, 59(3), 3042-3049.

Stefanics, G., Kremlacek, J., \& Czigler, I. (2014). Visual mismatch negativity: A predictive coding view. Frontiers in Human neuroscience, 8. doi:10.3389/fnhum.2014.00666

Sulykos, I. (2017). Visual mismatch negativity to elementary stimulus features (Doctoral thesis, Eötvös Loránd University, Budapest, Hungary).

Sulykos, I., \& Czigler, I. (2011). One plus one is less than two: Visual features elicit non-additive mismatch-related brain activity. Brain Research, 1398, 64-71.

Sulykos, I., Kecskés-Kovács, K., \& Czigler, I. (2015). Asymmetric effect of automatic deviant detection: The effect of familiarity in visual mismatch negativity. Brain Research, 1626, 108-117.

Susac, A., Heslenfeld, D., Huonker, R., \& Supek, S. (2013). Magnetic source localization of early visual mismatch response. Brain Topography. doi:10.1007/s10548-013-0340-8

Takács, E., Sulykos, I., Czigler, I., Barkaszi, I., \& Balázs, L. (2013). Oblique effect in visual mismatch negativity. Frontiers in Human Neuroscience, 7, 591.

Urakawa, T., Inui, K., Yamashiro, K., \& Kakigi, R. (2010). Cortical dynamics of the visual change detection process. Psychophsysiology, 47(5), 905-912. doi:10.1111/j.1469-8986. 2010.00987.x

Wang, X.-D., Liu, A.-P., Wu, Y.-Y., \& Wang, P. (2013). Rapid extraction of lexical tone phonology in Chinese characters: A visual mismatch negativity study. PLoS ONE, 8(2), e56778.

Wang, W., Miao, D., \& Zhao, L. (2014). Visual MMN elicited by orientation changes of faces. Journal of Integrative Neuroscience, 13(03), 485-495. doi:10.1142/S0219635214500137

Winkler, I., \& Czigler, I. (2012). Evidence from auditory and visual event-related potential (ERP) studies of deviance detection (MMN and $\mathrm{vMMN}$ ) linking predictive coding theories and perceptual object representations. International Journal of Psychophysiology, 82, 132-143.

Winkler, I., Karmos, G., \& Näätänen, R. (1996). Adaptive modeling of the unattended acoustic environment reflected in the mismatch negativity event-related potential. Brain Research, 742, 239-252.

Zhao, L., \& Li, J. (2006). Visual mismatch negativity elicited by facial expressions under non-attentional condition. Neuroscience Letters, 410(2), 126-131. 